\title{
CARACTERIZACIÓN MORFOAGRONÓMICA DE LA BERENJENA (Solanum melongena L.)
}

\section{MORPHOAGRONOMICAL CHARACTERIZATION OF AN EGGPLANT (Solanum melongena L.)}

\author{
Hermes Aramendiz-Tatis ${ }^{1}$, Cláudia Sudré2 ${ }^{2}$ Miguel Espitia ${ }^{1}$, Carlos Cardona ${ }^{3}$, Ender Correa ${ }^{4}$
}

${ }^{1}$ Ingeniero Agrónomo, Ph.D. Universidad de Córdoba, Departamento de Ingeniería Agronómica y Desarrollo Rural. Carrera 6 \# 76-103 Montería, Colombia. haramendiz@hotmail.com, ${ }^{2}$ Ingeniero Agrónomo,Ph.D. UENF, Av. Alberto Lamego, 2000, Parque Califórnia, 28013-602 Campos dos Goytacazes - RJ. Brasil. ${ }^{3}$ Ingeniero Agrónomo, M.Sc. Universidad de Córdoba. ceca24@hotmail.com, ${ }^{4}$ Ingeniero Agrónomo. Asistente de Investigación. endermanz@hotmail.com

Rev. U.D.C.A Act. E Div. Cient. 12 (2): 101-111, 2009

\section{RESUMEN}

El conocimiento de la diversidad genética presente en un banco de germoplasma es importante en el mejoramiento de plantas, porque permite identificar las combinaciones híbridas con mayor efecto heterótico y facilita la selección de segregantes transgresivos superiores en las poblaciones. El objetivo del trabajo consistió en estimar la divergencia genética entre 62 accesiones de Berenjena colectadas en el Caribe Colombiano, por la Universidad de Córdoba y Corpoica, mediante el uso de 21 caracteres morfoagronómicos. Los datos estuvieron constituidos por la moda de cada descriptor, de acuerdo a los criterios del IBPGR (1988). Se obtuvieron valores de similitud, discriminación de genotipos por el método de Cole Rodgers et al. (1997) y el de agrupación de pares no ponderados con medias aritméticas (UPGMA), utilizando el programa Genes (Cruz, 2006). Las características color del cotiledón, hábito de crecimiento de la planta, ancho de la hoja, lóbulos foliares, espinas en las hojas, pubescencia de la hoja, ancho del fruto y longitud del cáliz acusaron ausencia de variación y siete grupos fueron identificados, dos, de los cuales, integrados por un solo cultivar. Con base en el dendrograma y los descriptores utilizados los cultivares 48 y 56 son posibles duplicados.

Palabras clave: Análisis multivariado, recursos genéticos, banco de germoplasma.

\section{SUMMARY}

Knowing the genetic diversity of a gene bank is important in plant breeding because it allows the identification of hybrid combinations with greater heterotic effects and facilitates selection of superior transgressive segregates in populations. The objective of this study was to estimate the genetic divergence among 62 eggplant accessions collected in the Colombian Caribbean, using 21 morphoagronomical characters. The data were the mode for each descriptor according to IBPGR (1988). Genotype Similarity Discrimination values were obtained using the Cole Rodgers et al. (1997) method and the Unweighted Pair Group with Arithmetic Means (UPGMA) method using Genes (Cruz, 2006). Descriptors such as cotyledon color, growth habit, leaf width, leaf lobes, thorns on leaves, leaf pubescence and fruit calyx width and length presented lack of variation. Seven groups were identified, two of which comprise a single accession. Dendrogram showed that accessions identified as 48 and 56 were identical based on the descriptors.

Key words: Multivariate analysis, genetic resources, gene bank.

\section{INTRODUCCIÓN}

La berenjena fue introducida a Colombia desde España por los árabes en la década del 30 del siglo XIX y hoy 
en día, es la hortaliza de mayor importancia en los departamentos de Córdoba, de Sucre y de Bolívar, donde goza de gran demanda en la alimentación y por sus propiedades medicinales (Aramendiz et al. 2008). Su proceso de introducción fue mediante viajes de comercio que, poco a poco, se adaptó a las diferentes regiones. Estas poblaciones han estado sujetas a procesos de selección natural y artificial, mutaciones, deriva genética, migración que, asociados con la hibridación natural entomófila (Aramendiz et al. 2006; Gemmill-Herren $\varepsilon$ Ochieng, 2008), conducen a la recombinación y segregación, generando variabilidad genética (Prohens et al. 2005).

El conocimiento de la diversidad genética dentro y entre poblaciones vegetales, así como el tipo de variación que conduce a la formación de nuevas especies, es esencial en los estudios de evolución (Nei E Kumar, 2000). Así mismo, la divergencia genética es importante en el mejoramiento de plantas, ya que el uso racional de los recursos genéticos permite reducir los riesgos de vulnerabilidad a factores bióticos y abióticos, al igual que obtener progresos genéticos que conlleven al desarrollo de nuevos cultivares (Cui et al. 2001).

La evaluación de la divergencia genética de las poblaciones permite identificar las combinaciones híbridas de mayor efecto heterótico y mayor heterocigocidad, logrando la obtención de genotipos de combinaciones alélicas favorables o superiores en las generaciones segregantes. De esta manera, el estudio de la diversidad genética de las poblaciones, sienta las bases para la identificación de genotipos divergentes y auxilia al mejorador en la selección de combinaciones más promisorias y favorables a los cruzamientos (Falconer, 1989). El uso de padres con insuficiente divergencia genética en la formación de poblaciones segregantes, mediante la hibridación, reduce las posibilidades de encontrar genotipos de combinaciones genéticas favorables, dada la reducida variabilidad genética que ellos generan (Fehr, 1987).

La obtención de cultivares comerciales más productivos y de mejor calidad de fruto debe involucrar las características exigidas por productores y por consumidores de los mercados nacionales e internacionales, razón por la cual, el mejorador se constituye en un guardián de las características de mayor importancia en la comercialización del producto, como color, forma, ancho y tamaño del fruto (Sudré et al. 2006). En berenjena, los caracteres cualitativos como estrías, color, forma y tamaño del fruto son algunos de los caracteres importantes para el consumidor; en tanto, que el hábito de crecimiento de la planta, espinas en el cáliz del fruto, espinas foliares y peso de fruto, representan aspectos importantes para el productor.

Entre los métodos aplicados en el estudio de la divergencia genética, se destacan para variables cuantitativas la distancia Euclidiana y la distancia de Mahalanobis ( $\left.\mathrm{D}^{2}\right)$. Esta última considera las varianzas y las covarianzas residuales existentes entre las características medidas, cuando se establece el experimento bajo un diseño experimental; en tanto que la primera, se estima a partir de datos sin repeticiones (Cruz \& Carneiro, 2003). Para variables cualitativas, el índice de similitud (distancia de Cole Rodgers), representa el porcentaje de coincidencia de similitud, de un determinado valor, al considerar todos los caracteres analizados (Cole Rodgers et al. 1997). De esta manera, se obtiene la matriz de disimilitud a partir de dichos índices, para posteriormente realizar el análisis de agrupamiento (Coimbra et al. 2001; Cruz E Carneiro, 2003).

Abreu et al. (2002) estudiaron la divergencia genética entre 34 genotipos de tomate, utilizando características cualitativas. El análisis permitió separar los genotipos en diez grupos por el método de agrupamiento de Tocher (Rao, 1952), y las funciones discriminantes estimadas fueron eficaces en ubicar los genotipos en sus respectivas clases. De igual manera, Sudré et al. (2006) aplicaron esta técnica en 59 accesiones de ají y de pimentón, empleando 13 características y confirmaron la eficacia del agrupamiento de los genotipos, de acuerdo a la distancia genética.

El objetivo del trabajo fue evaluar la divergencia genética de 62 accesiones de berenjena colectadas en los departamentos de Córdoba, de Sucre y de Magdalena y conservado en el banco de germoplasma de C.I. "Turipaná" y Universidad de Córdoba, sobre la base descriptores morfoagronómicos, para la orientación de un programa de mejoramiento genético de la especie.

\section{MATERIALES Y MÉTODOS}

Sesenta y dos accesiones de berenjena del banco de germoplasma de la Universidad de Córdoba y Corpoica, colectadas en diferentes nichos de los departamentos 
de Córdoba, de Sucre y de Magdalena fueron utilizadas en este estudio (Tabla 1). El experimento fue realizado en Corpoica C.I. "Turipaná" de Cereté (Córdoba), en el primer semestre de 2008. Cada accesión fue sembrada en un área de $10 \mathrm{~m}^{2}$, en un surco de $10 \mathrm{~m}$ de longitud, con distancias entre plantas y surco de $1,0 \times 1,0 \mathrm{~m}$, sin repeticiones, para una población de once plantas por unidad experimental y un total de 62 unidades experimentales.

Los datos fueron obtenidos por medio de la moda de cada descriptor, de un total de once plantas en el surco de cada accesión. Fueron evaluadas las siguientes características: color del cotiledón, color del hipocótilo, hábito de crecimiento de la planta, ancho de la hoja, lóbulos de las hojas, espinas en las hojas, pubescencia de las hojas, número de flores por inflorescencia, color de la corola, longitud del fruto, ancho del fruto, relación longitud ancho del fruto, longitud del cáliz, espinas en el cáliz, estrías en el fruto, color del fruto, textura del fruto, forma del fruto, peso promedio de fruto, número de frutos por planta en cada cosecha y rendimiento, siguiendo los lineamientos del IBPGR (1988). Estas tres últimas características fueron categorizadas, para uniformizar todas las variables de respuesta.

El manejo agronómico, se realizó aplicando las recomendaciones dadas por Aramendiz et al. (2008) para el cultivo de la berenjena en el Valle del Sinú.

Tabla 1. Origen de las accesiones de berenjena colectadas en el Caribe Colombiano.

\begin{tabular}{|c|c|c|c|c|c|c|}
\hline \multirow{2}{*}{$\begin{array}{c}\text { GENOTIPO } \\
\text { No. }\end{array}$} & \multirow{2}{*}{$\begin{array}{l}\text { CÓDIGO } \\
\text { ACCESIÓN }\end{array}$} & \multirow{2}{*}{$\begin{array}{l}\text { NOMBRE COMÚN } \\
\text { DADO AL MATERIAL }\end{array}$} & \multirow{2}{*}{ MUNICIPIO } & \multirow{2}{*}{ SITIO DE COLECTA } & \multicolumn{2}{|c|}{ COORDENADAS } \\
\hline & & & & & Latitud & Longitud \\
\hline 1 & $\mathrm{C} 001$ & Negra & Cereté (C) & Mateo Gómez, Isla Blanca. & $085044 \mathrm{~N}$ & $755115 \mathrm{~W}$ \\
\hline 2 & $\mathrm{C} 002$ & Lila & Cereté $(\mathrm{C})$ & Mateo Gómez, Isla Blanca. & $085044 \mathrm{~N}$ & $755115 \mathrm{~W}$ \\
\hline 3 & $\mathrm{CO03}$ & Lila pompa & Cereté $(\mathrm{C})$ & Mateo Gómez, Isla Blanca. & $085039 \mathrm{~N}$ & $755115 \mathrm{~W}$ \\
\hline 4 & C004 & Morada & Cereté $(C)$ & $\begin{array}{l}\text { Mateo Gómez, Retiro de los } \\
\text { Páez }\end{array}$ & $085141 \mathrm{~N}$ & $755036 \mathrm{~W}$ \\
\hline 5 & C005 & Lila & Cereté $(C)$ & $\begin{array}{l}\text { Mateo Gómez, Retiro de los } \\
\text { Páez }\end{array}$ & $085141 \mathrm{~N}$ & $755036 \mathrm{~W}$ \\
\hline 6 & $\mathrm{C} 006$ & Negra & Cereté $(C)$ & $\begin{array}{l}\text { Mateo Gómez, Retiro de los } \\
\text { Páez }\end{array}$ & $085141 \mathrm{~N}$ & $755036 \mathrm{~W}$ \\
\hline 7 & $\mathrm{CO07}$ & Morada & Cereté $(\mathrm{C})$ & Mateo Gómez, Bergantín & $085036 \mathrm{~N}$ & $755039 \mathrm{~W}$ \\
\hline 8 & $\mathrm{CO08}$ & Negra & San Carlos (C) & Coroza San Carlos & $084805 \mathrm{~N}$ & $754606 \mathrm{~W}$ \\
\hline 9 & C009 & Morada & San Carlos (C) & Coroza San Carlos & $084805 \mathrm{~N}$ & $754606 \mathrm{~W}$ \\
\hline 10 & $\mathrm{C} 010$ & Morada larga & Lorica $(\mathrm{C})$ & La Doctrina, El Hierro & 091823N & $755418 \mathrm{~W}$ \\
\hline 11 & C011 & Lila larga delgada & Cereté $(\mathrm{C})$ & Retiro de los Indios & $085139 \mathrm{~N}$ & $754921 W$ \\
\hline 12 & $\mathrm{C} 012$ & Blanca & Cereté $(\mathrm{C})$ & Retiro de los Indios & $085139 \mathrm{~N}$ & $754921 W$ \\
\hline 13 & $\mathrm{C} 013$ & Lila color carne & Cereté $(\mathrm{C})$ & Retiro de los Indios & $085139 \mathrm{~N}$ & $754921 W$ \\
\hline 14 & C014 & Lila & Cereté $(\mathrm{C})$ & Retiro de los Indios & $085139 \mathrm{~N}$ & $754921 \mathrm{~W}$ \\
\hline 15 & $\mathrm{C} 015$ & Lila campana & Cereté $(C)$ & $\begin{array}{c}\text { Retiro de los Indios-Nuevo } \\
\text { Horizonte }\end{array}$ & $085105 N$ & $754835 W$ \\
\hline 16 & $\mathrm{C} 016$ & Lila & Cereté $(C)$ & $\begin{array}{c}\text { Retiro de los Indios-Nuevo } \\
\text { Horizonte }\end{array}$ & $085105 \mathrm{~N}$ & $754835 \mathrm{~W}$ \\
\hline 17 & $\mathrm{C} 017$ & Lila Clara & Cereté $(\mathrm{C})$ & Mateo Gómez & $085031 \mathrm{~N}$ & $754953 W$ \\
\hline 18 & $\mathrm{C} 018$ & Negra Linda & Cereté $(\mathrm{C})$ & Mateo Gómez & $085031 \mathrm{~N}$ & $754953 W$ \\
\hline 19 & $\mathrm{C} 019$ & Lila morada oscura & Cereté $(\mathrm{C})$ & Mateo Gómez & $085031 \mathrm{~N}$ & $754953 \mathrm{~W}$ \\
\hline 20 & $\mathrm{CO} 20$ & Morada & Cereté $(\mathrm{C})$ & La Coroza Argentina & $084842 \mathrm{~N}$ & $754514 W$ \\
\hline 21 & $\mathrm{C} 021$ & Negra & Cereté (C) & La Coroza Argentina & $084842 \mathrm{~N}$ & $754514 W$ \\
\hline 22 & $\mathrm{CO} 22$ & Lila & Cereté $(\mathrm{C})$ & Los Venados-Campanito & $084913 \mathrm{~N}$ & $754624 W$ \\
\hline 23 & $\mathrm{C} 023$ & Berenjena Palanca & Cereté $(\mathrm{C})$ & Retiro de los Indios & $085139 \mathrm{~N}$ & $754921 W$ \\
\hline
\end{tabular}


Continuación tabla 1 .

\begin{tabular}{|c|c|c|c|c|c|c|}
\hline \multirow{2}{*}{$\begin{array}{c}\text { GENOTIPO } \\
\text { No. }\end{array}$} & \multirow{2}{*}{$\begin{array}{l}\text { CÓDIGO } \\
\text { ACCESIÓN }\end{array}$} & \multirow{2}{*}{$\begin{array}{l}\text { NOMBRE COMUN } \\
\text { DADO AL MATERIAL }\end{array}$} & \multirow{2}{*}{ MUNICIPIO } & \multirow{2}{*}{ SITIO DE COLECTA } & \multicolumn{2}{|c|}{ COORDENADAS } \\
\hline & & & & & Latitud & Longitud \\
\hline 24 & $\mathrm{C} 024$ & Lila morada & Cereté $(\mathrm{C})$ & Retiro de los Indios & $085115 \mathrm{~N}$ & $754837 \mathrm{~W}$ \\
\hline 25 & $\mathrm{C} 025$ & Roja calabaza & Cereté $(\mathrm{C})$ & La Seibita 3 & $085041 \mathrm{~N}$ & $754442 W$ \\
\hline 26 & $\mathrm{C} 026$ & Morada & San Carlos (C) & El Tajo & $085130 \mathrm{~N}$ & $754332 W$ \\
\hline 27 & $\mathrm{C} 027$ & Negra & San Carlos (C) & El Tajo & $085130 \mathrm{~N}$ & $754332 W$ \\
\hline 28 & $\mathrm{C} 028$ & Lila & San Carlos (C) & El Tajo & $085130 \mathrm{~N}$ & $754332 W$ \\
\hline 29 & $\mathrm{C029}$ & Morada-Negra & San Carlos (C) & El Tajo & $085130 \mathrm{~N}$ & $754332 W$ \\
\hline 30 & $\mathrm{C030}$ & Negra larga & San Carlos (C) & El Tajo & $085130 \mathrm{~N}$ & $754332 W$ \\
\hline 31 & C031 & Verde & San Carlos (C) & El Tajo & $085130 \mathrm{~N}$ & $754332 W$ \\
\hline 32 & $\mathrm{C032}$ & Morada redonda & Lorica $(\mathrm{C})$ & La Doctrina-Hicotea & $091823 \mathrm{~N}$ & $755418 \mathrm{~W}$ \\
\hline 33 & $\mathrm{C} 033$ & Morada con espina & Lorica $(\mathrm{C})$ & La Doctrina-El Hierro & $091844 \mathrm{~N}$ & $755350 \mathrm{~W}$ \\
\hline 34 & $\mathrm{C} 034$ & Redonda & Lorica (C) & La Doctrina-El Hierro & $091844 \mathrm{~N}$ & $755350 \mathrm{~W}$ \\
\hline 35 & $\mathrm{C} 035$ & Morada con estrías & Lorica $(\mathrm{C})$ & La Doctrina-El Hierro & 091844N & $755350 \mathrm{~W}$ \\
\hline 36 & $\mathrm{C} 036$ & Negra bola de burro & Cereté $(\mathrm{C})$ & Los Venados-Campanito & $084913 N$ & $754624 W$ \\
\hline 37 & $\mathrm{C} 037$ & Negra-verde & Cereté $(\mathrm{C})$ & Los Venados-Campanito & $084913 N$ & $754624 \mathrm{~W}$ \\
\hline 38 & $\mathrm{C} 038$ & Morada & Cereté $(\mathrm{C})$ & Martínez-La Montañita & $085047 \mathrm{~N}$ & $754613 W$ \\
\hline 39 & $\mathrm{C} 039$ & Negra & Cereté $(\mathrm{C})$ & Martínez-La Montañita & $085047 \mathrm{~N}$ & $754613 W$ \\
\hline 40 & $\mathrm{C} 040$ & Morada & Cereté $(\mathrm{C})$ & La Pozona & $084951 \mathrm{~N}$ & $754708 W$ \\
\hline 41 & $\mathrm{C} 041$ & Negra & Cereté $(\mathrm{C})$ & La Pozona & $084951 \mathrm{~N}$ & $754708 W$ \\
\hline 42 & $\mathrm{C} 042$ & Negra redonda & Montería (C) & Las Babillas & $084814 \mathrm{~N}$ & $754747 \mathrm{~W}$ \\
\hline 43 & $\mathrm{C} 043$ & Negra larga & Montería (C) & Las Babillas & $084814 \mathrm{~N}$ & $754747 \mathrm{~W}$ \\
\hline 44 & $\mathrm{C} 044$ & Morada & Montería (C) & El Faro 2 & $084754 \mathrm{~N}$ & $754912 W$ \\
\hline 45 & $\mathrm{C} 045$ & Negra & Montería (C) & El Faro 2 & $084754 \mathrm{~N}$ & $754912 W$ \\
\hline 46 & $\mathrm{C} 046$ & Morada lila & Cereté $(\mathrm{C})$ & Mateo Gómez & $085343 \mathrm{~N}$ & $754903 W$ \\
\hline 47 & $\mathrm{C} 048$ & Morada larga gruesa & Cereté $(\mathrm{C})$ & El Quemao-Buenavista & $085213 \mathrm{~N}$ & $754911 W$ \\
\hline 48 & C049 & Morada gajito & Cereté $(\mathrm{C})$ & El Quemao-Buenavista & $085213 \mathrm{~N}$ & $754911 W$ \\
\hline 49 & $\mathrm{C} 050$ & Lila & Cereté $(\mathrm{C})$ & La Pozona & 084954N & $754620 \mathrm{~W}$ \\
\hline 50 & C051 & Morada & Montería (C) & Las Parcelas & $084338 \mathrm{~N}$ & $755458 w$ \\
\hline 51 & C052 & Morada & Montería (C) & Las Parcelas & $084311 \mathrm{~N}$ & $755553 \mathrm{~W}$ \\
\hline 52 & $\mathrm{C} 053$ & Morada & Montería (C) & Las Parcelas & $084311 \mathrm{~N}$ & $755553 w$ \\
\hline 53 & C054 & Morada & Cereté $(\mathrm{C})$ & Retiro de los Indios & $085138 \mathrm{~N}$ & $754920 w$ \\
\hline 54 & S001 & Cacho de chivo & Corozal (S) & El Rincón de las flores & $091605 \mathrm{~N}$ & $751828 W$ \\
\hline 55 & S002 & Pompa & Corozal (S) & El Rincón de las flores & $091605 \mathrm{~N}$ & $751828 W$ \\
\hline 56 & S003 & Pompa & Corozal (S) & El Rincón de las flores & $091605 \mathrm{~N}$ & $751828 W$ \\
\hline 57 & S004 & Cacho de chivo & Corozal (S) & El Rincón de las flores & $091605 \mathrm{~N}$ & $751828 W$ \\
\hline 58 & S005 & Morada con estría & Corozal (S) & El Rincón de las flores & $091605 \mathrm{~N}$ & $751828 W$ \\
\hline 59 & S006 & Lila delgada & Corozal (S) & El Guaimaro & 091252N & $752511 W$ \\
\hline 60 & M002 & Morada & Sitio Nuevo (M) & Carmona & $104823 N$ & $744679 W$ \\
\hline 61 & M003 & Morada & Sitio Nuevo (M) & Carmona & $104929 \mathrm{~N}$ & $744353 W$ \\
\hline 62 & M004 & Morada & Sitio Nuevo (M) & Carmona & $104929 \mathrm{~N}$ & $744353 \mathrm{~W}$ \\
\hline
\end{tabular}

C: Córdoba; S: Sucre; M: Magdalena 
El análisis estadístico consistió en la obtención de una matriz de disimilitud con las variables cualitativas y cuantitativas categorizadas. Esta metodología consiste en la obtención de un índice, en el cual, son considerados varios caracteres simultáneamente, de tal manera que cada característica puede presentar varias clases. Este índice contempla la coincidencia y no coincidencia de valores.

La distancia entre los genotipos i y j, sin considerar el número de caracteres o clases participantes, esta dada por la fórmula:

$$
\begin{gathered}
D_{i j}=1-\frac{c}{c+d} \\
c=\text { Coincidencia de valores } \\
d=\text { No coincidenaia de valores }
\end{gathered}
$$

Con los valores de disimilitud fue realizada la construcción del dendrograma por el método de agrupación de pares no ponderados con medias aritméticas (UPGMA) y fue evaluada la consistencia del agrupamiento por la correlación cofenética, utilizando el programa Genes (Cruz, 2006).

\section{RESULTADOS Y DISCUSIÓN}

El aprovechamiento de los recursos filogenéticos esta en función de la variabilidad genética de las accesiones presentes en un banco de germoplasma. En este sentido, los resultados encontrados señalan la existencia de polimorfismo en algunos de los caracteres considerados, lo que coadyuva a la selección de genotipos divergentes con elevado potencial heterótico, para la conformación de poblaciones con atributos agronómicos deseables por productores y por consumidores.

Los valores de similitud encontrados para los 21 caracteres oscilaron entre 0,48 y 1,0 (Tabla 2), destacando que el $50 \%$ de los genotipos del banco de germoplasma regional resultaron no coincidentes en diez o menos características estudiadas, lo que corrobora lo anotado por Prohens et al. (2005) y Hoyos et al. (1999), quienes anotan que es posible por el proceso de hibridación y recombinación genética, con posterior selección natural y artificial, con adaptación a condiciones ambientales de los Valles del Sinú y sabanas de Sucre y de Bolívar, ya que en las áreas productoras es posible encontrar ecotipos de Solanum torvum Sw, lo que posibilita el flujo genético entre la especie silvestre y la domesticada.

Las accesiones 48 y 51 resultaron coincidentes en todas las características y algunos genotipos, como el 46 y 47; 18 y 42; 16 y 60, al igual que 5 y 24, en 20 de los caracteres considerados. Las características que presentaron ausencia de variación corresponden a color del cotiledón, hábito de crecimiento de la planta, ancho de la hoja, lóbulos foliares, espinas en las hojas, pubescencia de la hoja, ancho del fruto y longitud del cáliz; tal comportamiento refleja poco polimorfismo genético entre los genotipos estudiados y evidencia que dichas accesiones no acusan una marcada divergencia genética respecto al material original, sugiriendo la existencia de una base genética estrecha para dichos caracteres, como lo señalan Karihaloo et al. (1995), constituyendo posibles duplicados del material genético por la distribución antrópica, lo cual, debe ser corroborado mediante marcadores moleculares (Behera et al. 2006), y demuestra que el proceso evolutivo en dichos recursos genéticos es lento, ya que las accesiones provienen de un mismo ancestral y confirma que la distancia geográfica no siempre está correlacionada con la diversidad genética, como lo encontraron Huh E Huh (2001), en lenteja (Vicia faba L.) y Nascimento Filho et al. (2001), en guaraná (Paullinia cupana L.). En este sentido, la diversidad geográfica no puede ser utilizada como indicador de diversidad genética en un programa de mejoramiento para conformar poblaciones segregantes, como algunas veces es realizado (Cruz $\mathcal{E}$ Carneiro, 2003).

Al considerar la magnitud de los resultados existe riesgo en el uso de dichos recursos genéticos en los diferentes sistemas de producción, por la vulnerabilidad a factores bióticos y abióticos, dada la alta homogeneidad genética de los caracteres estudiados y el uso recurrente de los mismos materiales, por nuestros productores. Esto demanda la introgresión de nuevos genes, que permitan ampliar la base genética de las poblaciones para desarrollar nuevos cultivares, que respondan a las expectativas de productores y de consumidores. Sin embargo, los procesos de selección acusan la existencia de frutos de diferentes peso, forma, tamaño y color de fruto, lo que permite su uso de acuerdo a la divergencia genética, como posibles padres en un programa de mejoramiento, ya que además de ello, poseen una amplia adaptación y estabilidad a nuestras 
Tabla 2. Valores más destacados de caracteres coincidentes (CP), no coincidentes (D) y de similitud (V) para 21 caracteres considerados en el estudio de 62 cultivares de berenjena colectada en el Caribe Colombiano.

\begin{tabular}{|c|c|c|c|c|c|c|c|}
\hline GENOTIPOS & $\mathrm{CP}$ & D & V & GENOTIPOS & $\mathrm{CP}$ & D & V \\
\hline $48-51$ & 21 & 0 & 1 & $29-30$ & 19 & 2 & 0,904761 \\
\hline $5-4$ & 20 & 1 & 0,952381 & $35-57$ & 19 & 2 & 0,904761 \\
\hline $16-60$ & 20 & 1 & 0,952381 & $36-41$ & 19 & 2 & 0,904761 \\
\hline $18-42$ & 20 & 1 & 0,952381 & $43-56$ & 19 & 2 & 0,904761 \\
\hline $46-47$ & 20 & 1 & 0,952381 & $44-54$ & 19 & 2 & 0,904761 \\
\hline $1-9$ & 19 & 2 & 0,904761 & $48-57$ & 19 & 2 & 0,904761 \\
\hline $2-42$ & 19 & 2 & 0,904761 & $51-57$ & 19 & 2 & 0,904761 \\
\hline $3-48$ & 19 & 2 & 0,904761 & $54-56$ & 19 & 2 & 0,904761 \\
\hline $3-51$ & 19 & 2 & 0,904761 & $55-56$ & 19 & 2 & 0,904761 \\
\hline $4-29$ & 19 & 2 & 0,904761 & $57-59$ & 19 & 2 & 0,904761 \\
\hline $5-18$ & 19 & 2 & 0,904761 & $2-23$ & 10 & 11 & 0,47619 \\
\hline $5-28$ & 19 & 2 & 0,904761 & $3-37$ & 10 & 11 & 0,47619 \\
\hline $6-36$ & 19 & 2 & 0,904761 & $4-45$ & 10 & 11 & 0,47619 \\
\hline 6-39 & 19 & 2 & 0,904761 & $8-12$ & 10 & 11 & 0,47619 \\
\hline $6-41$ & 19 & 2 & 0,904761 & $12-22$ & 10 & 11 & 0,47619 \\
\hline 7-15 & 19 & 2 & 0,904761 & $12-36$ & 10 & 11 & 0,47619 \\
\hline $7-24$ & 19 & 2 & 0,904761 & $12-38$ & 10 & 11 & 0,47619 \\
\hline $7-26$ & 19 & 2 & 0,904761 & $12-39$ & 10 & 11 & 0,47619 \\
\hline $8-39$ & 19 & 2 & 0,904761 & $16-58$ & 10 & 11 & 0,47619 \\
\hline $10-15$ & 19 & 2 & 0,904761 & $20-30$ & 10 & 11 & 0,47619 \\
\hline $13-14$ & 19 & 2 & 0,904761 & $20-46$ & 10 & 11 & 0,47619 \\
\hline $13-48$ & 19 & 2 & 0,904761 & $22-52$ & 10 & 11 & 0,47619 \\
\hline $13-51$ & 19 & 2 & 0,904761 & $22-53$ & 10 & 11 & 0,47619 \\
\hline $14-17$ & 19 & 2 & 0,904761 & $25-52$ & 10 & 11 & 0,47619 \\
\hline $15-24$ & 19 & 2 & 0,904761 & $27-49$ & 10 & 11 & 0,47619 \\
\hline $15-33$ & 19 & 2 & 0,904761 & $27-53$ & 10 & 11 & 0,47619 \\
\hline $18-21$ & 19 & 2 & 0,904761 & $30-58$ & 10 & 11 & 0,47619 \\
\hline 18 - 24 & 19 & 2 & 0,904761 & $34-39$ & 10 & 11 & 0,47619 \\
\hline $24-57$ & 19 & 2 & 0,904761 & $37-52$ & 10 & 11 & 0,47619 \\
\hline $26-40$ & 19 & 2 & 0,904761 & $41-52$ & 10 & 11 & 0,47619 \\
\hline $28-57$ & 19 & 2 & 0,904761 & $58-60$ & 10 & 11 & 0,47619 \\
\hline
\end{tabular}


zonas productoras. De igual manera, los más divergentes deben ser evaluados por su capacidad de combinación general y específica, puesto que provienen de áreas de polinización abierta y sólo se considera el efecto materno.

El dendrograma obtenido por el método UPGMA registró un coeficiente de correlación cofenético de $(\mathrm{r}=0,68), \mathrm{p}<0,01$, lo que demuestra una moderada representatividad del conjunto de los datos de la matriz de similitud y la matriz original en el análisis de agrupamiento. Los 62 cultivares fueron agrupados en siete grupos (Figura 1): el primero, conformado por la accesión 58, registró como características divergentes cuatro flores por inflorescencia, pesos de fruto entre 176 y $217 \mathrm{~g}$ y rendimiento entre 12,3 y $18,3 \mathrm{t} \cdot \mathrm{ha}^{-1}$. Es la más disímil y no deseable, dada la presencia de más de una flor por cojín floral, ya que la planta por su naturaleza andromonóica, solo la flor principal es funcional para la formación de frutos, puesto que las de estilo corto actúan como masculinas, en razón a su estigma de tamaño pequeño, papilas poco desarrolladas y bajo contenido de azúcar (Rylski et al. 1984).

El segundo grupo integrado por 15 genotipos que representan el $24,19 \%$ de las accesiones del banco de germoplasma, presentan como características divergentes la presencia de frutos tres veces más largos que anchos y textura rugosa de los mismos, con tres frutos por planta y un peso que oscila entre 302 y $343 \mathrm{~g}$,

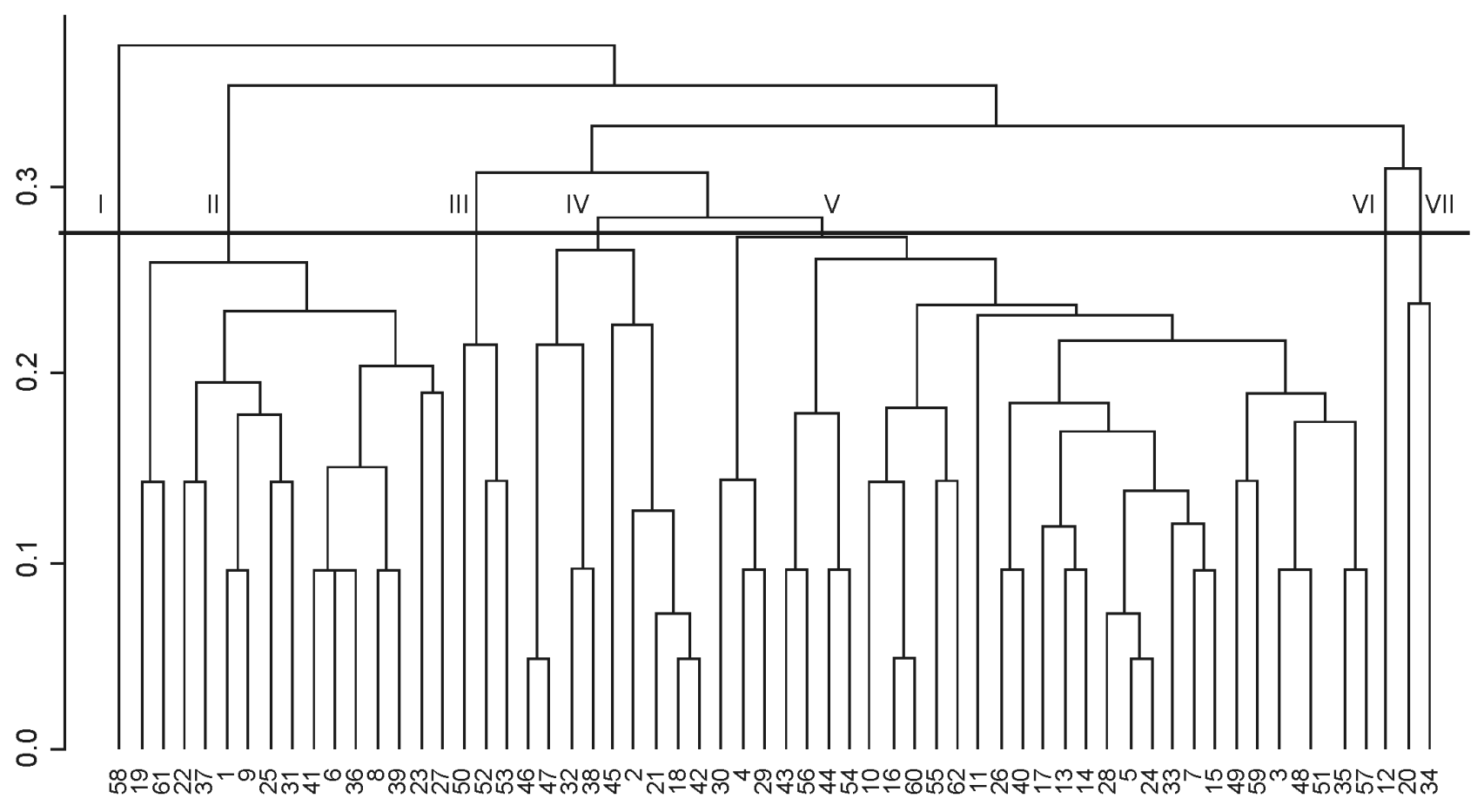

Figura 1. Dendrograma obtenido partir de 21 caracteres morfoagronómicos de berenjena, evaluados en 62 cultivares colectados en la región Caribe Colombiano, utilizando el método UP.

con rendimiento superior a $36 \mathrm{t} \cdot \mathrm{ha}^{-1}$. Es de gran interés este grupo por la capacidad o tolerancia de formar frutos bajo condiciones de altas temperaturas que inducen esterilidad del polen (Sanwal et al. 1997; Sasaki et al. 2005), lo que se traduce en una reducción en la formación de frutos (Nkansah, 2001) y, por lo tanto, pueden ser usados en un programa de mejoramiento genético como parentales, dada su adaptabilidad a condiciones tropicales, para formar poblaciones de buen rendimiento y calidad de fruto, de acuerdo a la demanda de productores (Kikuchi et al. 2008). Así mismo, el hecho de poseer frutos muchos más largos que anchos, 
los hace menos propensos a la pérdidas de agua por transpiración de fruto, ya que esta declina en función del área cubierta por el cáliz y la relación superficie del área y masa del fruto (Díaz-Pérez, 1998).

El grupo tres, compuesto por los genotipos 50, 52 y 53, muestra características sobresalientes, representadas por el color de hipocótilo violeta, presencia de tres flores por inflorescencia, frutos color lila de textura suave, peso de fruto entre 176 y $217 \mathrm{~g}$ y rendimiento entre 18,4 y $24,4 \mathrm{t} \cdot \mathrm{ha}^{-1}$.

El cuarto, integrado por las accesiones 2, 18, 21, 32, 38, 42, 45, 46 y 47; donde sobresale la presencia de hipocótilo de color verde, dos frutos por planta y rendimiento entre 24,5 y 30,5t $\cdot$ ha $^{-1}$. Desde el punto de vista económico, el uso de genotipos de este grupo puede ser deseable, en la obtención de híbridos que combinen una o más características asociadas con el rendimiento y la calidad de fruto, especialmente del grupo dos, como lo destacan Ingale et al. (1997), quienes reportaron heterosis en 45 híbridos, con respecto a sus padres.

El grupo cinco, conformado por el $50 \%$ de los genotipos, posee como características divergentes la presencia de frutos de color lila, con textura suave y tres frutos por planta. Considerando que el número de frutos por planta es un componente importante del rendimiento y dado que el color de fruto lila tiene un mercado de alta demanda en la región caribe, la hibridación entre genotipos de este grupo con cultivares del grupo tres, seguido de recombinación es una buena opción, ya que los efectos genéticos aditivos y no aditivos son influyentes en el rendimiento, lo que permite incrementar la posibilidad de seleccionar genotipos superiores por acumulación de alelos favorables, tanto para rendimiento como para calidad de fruto (Ahmed et al. 2006).

En el sexto grupo, conformado por el cultivar 12, se caracteriza por tener hipocótilo de color verde, cuatro flores por inflorescencia, frutos de color blanco y alargados, con pesos entre 218 y $259 \mathrm{~g}$, tres frutos por planta y rendimiento entre 12,3 y $18,3 \mathrm{t} \cdot \mathrm{ha}^{-1}$. Estos frutos por su color blanco carecen de niveles altos de flavonoides, que son una fuente importante de radicales libres para reducir los problemas de cáncer y de arteriosclerosis (Stommel \& Whitaker, 2003). La presencia de alto número de flores por inflorescencia es un carácter dominante y, por lo tanto, genotipos de este y otros grupos con alto número de flores, no son deseables, puesto que afecta la formación de frutos (Bletsos et al. 2004).

El grupo siete, integrado por los genotipos 20 y 24, se caracteriza por tener cuatro flores por inflorescencia y frutos dos veces más largos que ancho, de color morado. La intensidad, la uniformidad y el brillo del color de los frutos de los cultivares de este grupo, los tornan atractivo en un programa de mejoramiento genético, por su alto contenido de antocianina (Nothmann et al. 1976), que por su alto contenido de polifenoles reducen la cantidad de radicales libres y con ello, los problemas de enfermedades (Stommel \& Whitaker, 2003).

La agrupación de los cultivares corrobora lo encontrado por Peter \& Rai (1976), en tomate; Carvalho et al. (2003), en algodón y Prohens et al. (2005) en berenjena, quienes encontraron baja correlación entre la divergencia genética y el origen geográfico, ya que es posible encontrar cultivares oriundos de diferentes regiones conformando un mismo grupo; en tanto que genotipos, de una misma región, son clasificados en grupos diferentes. Estos resultados concuerdan con lo reportado por Furini \& Wunder (2004), quienes señalan que el movimiento de accesiones de un ambiente a otro crea confusión en torno su origen geográfico.

En un programa de mejoramiento genético, orientado al desarrollo de cultivares adaptados a las condiciones productoras del Caribe Colombiano, es importante la hibridación entre cultivares de mayor divergencia genética, ya que está relacionada con la heterosis (Gaur et al. 1978; Coimbra et al. 1978), lo que permite encontrar segregantes transgresivos que, además de su adaptación, poseen atributos agronómicos y de calidad de fruto, apetecidos por productores y por consumidores.

Las características de menor contribución a la divergencia genética y que representan una base genética estrecha fueron: color del cotiledón, hábito de crecimiento, ancho de la hoja, lóbulos foliares, espinas en las hojas, pubescencia de la hoja, ancho del fruto y longitud del cáliz, por tanto, su mejoramiento genético implica la introgresión de genes de poblaciones mejoradas.

Hibridaciones entre accesiones del grupo dos y cuatro podrán proporcionar segregantes transgresivos en 
generaciones avanzadas, a fin de obtener genotipos acordes a las demanda del mercado.

La cuantificación de la divergencia genética permitió identificar siete grupos, con la presencia de dos de ellos con un solo cultivar, corroborando la presencia de polimorfismo en los caracteres cualitativos e independencia en cuanto a su origen.

Se encontró coincidencia en la información entre las accesiones 48 y 51.

Finalmente, se considera que estudios de caracterización molecular a las accesiones del banco de germoplasma de berenjena, constituirían un gran soporte en el conocimiento de la divergencia genética del germoplasma colectado.

AGRADECIMIENTOS. A los productores de berenjena del caribe húmedo, Corpoica y la Universidad de Córdoba, por facilitar sus instalaciones para realizar esta investigación. Conflictos de intereses: El manuscrito fue preparado y revisado con la participación de todos los autores, quienes declaramos que no existe ningún conflicto de intereses que ponga en riesgo la validez de los resultados presentados. Financiación: Este estudio fue financiado por el Ministerio de Agricultura y Desarrollo Rural, Corpoica y la Universidad de Córdoba.

\section{BIBLIOGRAFÍA}

1. ABREU, F.B.; MARIM, B.G.; SILVA, D.J. H; GUIMARAES, M.A.; LUCA, C.A.; FAGUNDES, R. 2002. Determinação da divergência entre acessos de tomateiro mediante a utilizacao de variáveis multicategóricas.In: 42a Congresso Brasileiro de Olericultura. Uberlandia. Horticultura Brasileira (Brasil) CD ROM. 20(2).

2. AHMED, N.; MEHDI, M.; NAYEEMA, J.; SINH, A.K. 2006. Inheritance of quantitative characters in eggplant (Solanum melongena L.). Indian J. Agricult. 63(2):218-220.

3. ARAMENDIZ, H.; CARDONA, C.; JARMA, A.; ESPITIA, M. 2008. El cultivo de la berenjena (Solanum melongena L.). Universidad de Córdoba. Ed. Produmedios, Bogotá. 151p.
4. ARAMENDIZ, H.; CARDONA, C.; ROBLES, J.; FERNÁNDIEZ, C.; HERNÁNDEZ, J. 2006. Polinización cruzada natural en berenjena (Solanum melongena L.). Fitotecnia Colombiana. 6(1):59-66.

5. BEHERA, T.K.; SHARMA, P.; SING, B.K., KUMAR, G.; KUMAR, R.; MOHAPATRA, T., SING, N.K. 2006. Assessment of genetic diversity and relationships in eggplant (Solanum melongena L.) using STMs markers. Scientia Horticulturae (USA). 107(4):352357.

6. BLETSOS, F.; ROUPAKIAS, D.; TSAKTSIRA, M.; SCALTSOYJANNES, A. 2004. Production and characterization of interspecific hybrids between three eggplant (Solanum melongena L.) cultivars and Solanum macrocarpum L. Scientia Horticulturae. 101 (1):11-21.

7. CARVALHO, L.P.; ABREU, M.; FALLIERI, J.; SANTOS, J. 2003. Análise da diversidade genética entre acessos de banco ativo de germoplasma de algodão. Pesquisa Agropecuária Brasileira 38(10):1149-1155.

8. COIMBRA, R.R.; MIRANDA, G.V.; MOREIRA, G.R.; SILVA, D.J.; CRUZ, C.; CARNEIRO, P.; SOUZA, L.; GUIMARÃES, L.; MARCASSO, R.; CANIATO, F. 2001. Divergência genética de cultivares de milho baseada em descritores qualitativos. III SIRGEALC, p.266-268.

9. COIMBRA, J.L.; CARVALHO, F.I.; HEMP, S.; OLIVEIRA, A.C.; SILVA, S. 1978. Divergência genética em feijão preto. Ciência Rural (Brasil). 29(3):427-431.

10. COLE-RODGERS, P.; SMITH, D.W.; BOSLAND, P. W. 1997. A novel statistical approach to analyze genetic resources evolutions using Capsicum as an example. Crop Science (USA). 37(3):1000-1002.

11. CRUZ, C. 2006. Programa Genes. Versão Windows. Universidade Federal de Viçosa. 648p.

12. CRUZ, C.D.; CARNEIRO, P.C. 2003. Modelos biométricos aplicados ao melhoramento genético. Viçosa:UFV, 585p. 
13. CUI, Z.; CARTER, T.; BURTON, J.; WELLS, R. 2001. Phenotypic diversity of modern Chinese and North American soybean. Crop Science. 41(6):19541967.

14. DÍAZ-PÉREZ, J.C. 1998. Transpiration rates in eggplant fruit as affected by fruit and calyx size. Postharvest Biology and Technology (Nueva Zelandia ). 13 (1):45-49.

15. FALCONER, D.S. 1989. Introduction to quantitative genetics. 3.ed. New York, Logman, 489p.

16. FEHR, W.R. 1987. Principles of cultivar development. New York: Macmillan. 1: 536p.

17. FURINI, A.; WUNDER, J. 2004. Analysis of eggplant (Solanum melongena L.) related germplasm: morphological and AFLP data contribute to phylogenetic interpretations and germplasm utilization. Theoretical and Applied Genetic (Alemania ). 108(1):197-208.

18. GAUR, P.C.; GUPTA, P.K.; KISHORE, H. 1978. Studies on genetic divergence in potato. Euphytica (Holanda). 27(2):361-368.

19. GEMMILL-HERREN, B.; OCHIENG, A. 2008. Role of native bees and natural habitats in eggplant (Solanum melongena) pollination in Kenya. Agricultulture, Ecosystems $\&$ Environment (USA). 127(1):31-36.

20. HOYOS, F.; GARCIA, E.; ARAMENDIZ, H. 1999. Estimación de la variabilidad genética en una población criolla de berenjena. Revista Temas Agrarios (Colombia). 8:117-126.

21. HUH, M.K.; HUH, H.W. 2001. Genetic diversity and population structure of wild lentil tare. Crop Science. 41(6):1940-1946.

22. IBPGR. 1988. Descriptors for eggplant. International Board for Plant Genetic Resources, Rome. 23p.

23. INGALE, B.V.; PATIL, S.J.; BASARKAR, P.W. 1997. Heterosis for biochemical composition of fruits in eggplant (Solanum melongena L.). Indian J. Horticult. 54(4):327-332.
24. KARIHALOO, J.L.; BRAUNER, S.; GOTTLIEB, L.D. 1995. Ramdon amplified polymorphic DNA variation in the eggplant, Solanum melongena $\mathrm{L}$. (Solanaceae). Theor. Appl. Genetics (Alemania). 90(6):767-770.

25. KIKUCHI, K.; HONDA, I., MATSUO, S.; FUKUDA, M.; SAITO, T. 2008. Stability of fruit set newly selected parthenocarpic eggplant lines. Scientia Horticulturae.115(2):111-116.

26. NASCIMENTO FILHO, F.; ATROCH, A.; SOUSA, N.; GARCÍA, T.; CRAVO, M.; COUTINHO, E. 2001. Divergência genética entre clones de guaranazeiro. Pesquisa Agropecuária Brasileira. 36(3):501506.

27. NEI, M.; KUMAR, A. 2000. Molecular Evolution and Phylogenetics. Oxford University Press Inc. New York. 333p.

28. NKANSAH, G.O. 2001.Some physiological features of the African eggplant, Solanum aethiopicum group 'Gilo'. Scientia Horticulturae. 90 (1-2):181186.

29. NOTHMANN, J.; RYLSKI, I.; SPIGELMAN, M. 1976. Color and variations in color intensity of fruit of eggplant cultivars. Scientia Horticulturae. 4(2):191-197.

30. PETER, K.V.; RAI, B. 1976. Genetic divergence in tomato. The Indian J. Genetics and Plant Breeding. 32(3):907-912.

31. PROHENS, J.; BLANCA, J.M.; NUEZ, F. 2005. Morphological and Molecular Variation in a Collection of Eggplants from a Secondary Center of Diversity: Implications for Conservation and Breeding. J. Amer. Soc. Hort. Sci. (USA). 130 (1):54-63.

32. RAO, R.C. 1952. Adavances statiscal methods in biometric research. New York.Jhon Wiley and Sons, 330p.

33. RYLSKI, L.; NOTHMANN, J.; ARCAN, L. 1984. Differential fertility in short-sttyled eggplant flowers. Scientia Horticulturae. 22(1):39-46. 
34. SANWAL, S.K.; BASWANA, K.S.; DINGRA,H.R. 1997. High temperature tolerance in eggplant:stigma, anther and pollen studies. Ann Biol. (India).13(1):123125.

35. SASAKI, H.; YANO, T.; YAMASAKI, A. 2005. Reduction of high temperatura inhibition in tomato fruit set by plant growth regulators JARQ (Japón).39(2):135-138.

36. STOMMEL, J.R.; WHITAKER, B. 2003. Phenolic acid content and comosition of eggplant fruit in a germplasm core subset. J. Am. Soc. Hort. Sci. 128(5):704-710.
37. SUDRÉ, C.P.; CRUZ, C.; RODRIGUES, R.; RIVA, E.M.; AMARAL JÚNIOR, A.T.; SILVA, D.J.H.; PEREIRA, T.N. 2006. Variáveis multicategóricas na determinação da divergência genética entre acessos de pimenta e pimentão. Hort. Brasileira. 24(1):88-93.

Recibido: Diciembre 3 de 2008

Aceptado: Septiembre 1 de 2009 